\title{
Case Report of Exercise to Attenuate Side Effects of Treatment for Pancreatic Cancer
}

\author{
Marie McLaughlin ${ }^{a} \quad$ Alan Christie $^{b} \quad$ Anna Campbell ${ }^{a}$ \\ aEdinburgh Napier University, Edinburgh, UK; ${ }^{b}$ Edinburgh Cancer Centre, Western General \\ Hospital, Edinburgh, UK
}

\section{Keywords}

Pancreatic cancer · Exercise - Chemotherapy · Case report

\begin{abstract}
Treatments for pancreatic cancer can have debilitating side effects including fatigue, weight loss, and cardiac toxicity, resulting in functional loss and psychological distress. Exercise has been proposed as a therapy to counteract physical and psychological detriments. The case: A 47-year-old male undergoing chemotherapy for stage 3 locally advanced pancreatic cancer. He was cycling during hospital chemotherapy infusions ( 6 fortnightly cycles of FOLFIRINOX: 5FU 2, $400 \mathrm{mg} / \mathrm{m}^{2}$, over $48 \mathrm{~h}$ : irinotecan $180 \mathrm{mg} / \mathrm{m}^{2}$, oxaliplatin $85 \mathrm{mg} / \mathrm{m}^{2}$, no 5 -FU bolus) plus 12 weeks of twice weekly aerobic and resistance exercise. Over 12 weeks, body composition was maintained, and physical function improved, with specific increases in muscular strength of up to $50 \%$ and aerobic capacity improving by $9 \%$. Moreover, quality of life, fatigue, psychological distress, and sleep quality improved by $38,113,50$, and $9 \%$, respectively. Additionally, the participant experienced more severe side effects in week 6 , when he did not cycle to a high intensity during hospital infusion and had less total weekly exercise. After cycle 6 (week 11), chemotherapy was halted, and a Whipple resection procedure was successfully performed. It can be concluded that regular aerobic and resistance exercise plus exercise during infusion can
\end{abstract}




\section{Case Reports in Oncology}

Case Rep Oncol 2019;12:845-854

DOI: $10.1159 / 000503815$

2019 The Author(s). Published by S. Karger AG, Basel www.karger.com/cro

McLaughlin et al.: Case Report of Exercise to Attenuate Side Effects of Treatment for Pancreatic Cancer

attenuate expected decline in physical and mental health with pancreatic cancer treatment and may reduce treatment side effects and have favourable effects on prognosis.

(C) 2019 The Author(s)

Published by S. Karger AG, Basel

\section{Introduction}

The prognosis for pancreatic cancer is poor, with $<5 \%$ of those with the condition surviving for 5 years and only $1 \%$ surviving 10 years after diagnosis [1]. For resectable pancreatic cancer (stage 1 and 2) survival rates are $7-25 \%$ at 5 years. Locally advanced (stage 3 ) and advanced cancer (stage 4) have an average survival of 6-11 months and 2-6 months, respectively [1]. In addition to the poor prognosis, treatments for pancreatic cancer (primarily surgery, chemotherapy, and radiotherapy) can have debilitating side effects, including fatigue which affects $92 \%$ of patients receiving chemotherapy [2]. Further complications with pancreatic cancer include weight loss, cardiac toxicity, digestive problems, diarrhoea, nausea, vomiting, increased infection risk, alopecia, and pain due to chemotherapy treatment [3]. Additionally, psychological well-being decreases by $13 \%$ from pre- to post-treatment, with pancreatic cancer eliciting the highest rates of depression and anxiety compared to all other cancer types at 28.8 versus $18.5 \%$, respectively [4]. Furthermore, disturbed sleep is also common in cancer survivors, with $43 \%$ of cancer patients having insomnia syndrome during chemotherapy treatment [5]. This translates to a decrease in quality of life (QOL) with FACT-Hep scores declining from pre- to post-treatment in pancreatic cancer patients [3]. Therefore, therapies that improve treatment tolerance, reduce the loss of physical functioning, and improve psychological well-being are essential to improve QOL.

Exercise has been proposed as such a therapy and has been investigated in other cancer types. As current, only one single case study exists which investigates the effects of exercise on physical and psychological well-being in pancreatic cancer [6]. This previous study investigated twice weekly aerobic and resistance training for 6 months in a 49-year-old male with stage $2 \mathrm{~b}$ pancreatic cancer undergoing chemotherapy. The intervention was well-tolerated, with $73 \%$ attendance. At 12-weeks, exercise resulted in an improvement across all outcome measures, with an $8.2 \%$ increase in fat free mass, accompanied by a $31.6 \%$ increase in leg strength, and a decrease in time to complete $400 \mathrm{~m}$ walk, chair rise, stair climb, and $6 \mathrm{~m}$ walk $(-5.9,-17.2,-9.9$, and $-23.5 \%$, respectively). In addition to this, QOL improved by $40 \%$, fatigue improved by $350 \%$, sleep quality improved by $20 \%$, and psychological distress improved by $21 \%$. The current study was undertaken to expand these findings by investigating exercise during chemotherapy infusion in addition to twice weekly aerobic and strength exercise in a case of stage 3 pancreatic cancer.

\section{Presenting Concerns}

A 47-year old white male with diagnosis of stage 3 locally advanced pancreatic cancer was recruited following a self-referral to MoveMore cancer exercise classes. He is married, a nonsmoker, and works in middle management. He initially presented with a 2-3 month history of epigastric pains, requiring hospital admission on one occasion. After symptoms failed to settle 
with analgesia and omeprazole, he underwent further investigation with an endoscopic ultrasound and CT scan of his abdomen.

\section{Clinical Findings}

He had no medical history of note, and was taking omeprazole, tramadol and ibuprofen for symptom relief. There were no relevant family history or examination findings and he was ECOG performance status 0 .

\section{Diagnostic Focus and Assessment}

Investigations confirmed the presence of a locally advanced pancreatic adenocarcinoma with invasion of the superior mesenteric vein, and proximity of the tumour to the hepatic artery and superior mesenteric artery. Baseline laboratory testing was unremarkable, with excellent liver and kidney function. As a consequence of the vascular involvement, he had an AJCC stage III pancreatic cancer which was considered inoperable.

\section{Therapeutic Focus}

The case received FOLFIRINOX (5-FU 2,400 mg/m² over $48 \mathrm{~h}$, irinotecan $180 \mathrm{mg} / \mathrm{m}^{2}$, oxaliplatin $85 \mathrm{mg} / \mathrm{m}^{2}$ - no 5 -FU bolus) via peripherally inserted catheter administered over 6 fortnightly cycles ( 12 weeks).

During hospital chemotherapy infusions, the participant cycled for $40 \mathrm{~min}$ at $60 \%$ of heart rate maximum, using Polar Heart Rate Monitor. In addition, a 12-week exercise program, beginning on week 2 of chemotherapy, was delivered as one-on-one sessions on two nonconsecutive days/week, supervised by the researcher based on the following exercise prescription:

- 5-min warm-up and cool-down: light aerobic and flexibility exercises.

- Resistance exercise: 8 exercises, performed for 3 sets of 12 repetitions at $60 \%$ of 1 repetition maximum (1-RM), based on 12-RM baseline strength testing (leg press, leg extension, leg curl, calf raise, hip abduction, rear kick, back extension and leg raises). The participant did not perform upper body exercises as peripherally inserted central catheter was present.

- Aerobic exercise: 15 min of continuous cycling on ergometer at $70 \%$ of maximum heart rate, based on maximal heart rate during baseline testing. Heart rate monitor worn throughout all sessions.

Intensity and duration attenuated based on the participant's health status. Therefore, adherence and compliance were recorded throughout.

Additional exercise out-with the program was advised, with the aim of accumulating 150$\mathrm{min} /$ week moderate intensity or 75-min vigorous intensity exercise in bouts of 10 -min or more. 


\section{Case Reports in Oncology}

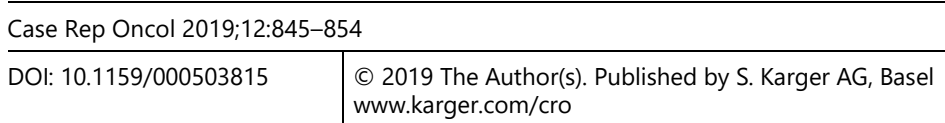

McLaughlin et al.: Case Report of Exercise to Attenuate Side Effects of Treatment for Pancreatic Cancer

\section{Assessments}

Aerobic Capacity: The participant cycled on an ergometer at 100 watts for 6 min. Heart rate measured every minute, using Polar heart rate monitor, and the steady state heart rate determined. This was extrapolated to corresponding $\mathrm{VO}_{2 \max }$ estimation on Astrand Ryhming nomogram.

Strength Testing (12-Repitition Maximum [RM]): The maximum load that can be performed for 12-repetition was determined for leg press, leg extension, calf raise, leg curl, rear kick, hip abduction, and back extension.

Flexibility: Seated toe-reach assessed flexibility of the hamstrings.

Physical Function: Repeated chair rise (time taken to rise five times from a chair) evaluated muscular power. Stair climb (time to ascend a flight of stairs) assessed ambulation. Usual pace, fast pace, and backwards 6-m walk evaluated ambulation and dynamic balance. Activities-Specific Balance Confidence scale evaluated falls self-efficacy.

Body Composition: Bioelectrical impedance analysis assessed percentage body fat and lean mass.

Physical Activity (PA) Levels: Apple watch assessed average weekly PA.

Questionnaires: The Functional Assessment of Cancer Therapy-Hepatobiliary (FACTHep) questionnaire evaluated pancreatic cancer-specific quality of life.

Functional Assessment of Chronic Illness Therapy-Fatigue subscale assessed cancer-related fatigue.

Pittsburgh Sleep Quality Index assessed sleep quality.

The Brief Symptom Inventory-18 assessed psychological distress (depression, anxiety, somatization, and global distress severity).

Semi-Structured Interview: Conducted at the end of the exercise program to elucidate if exercising during chemotherapy was truly meaningful. This consisted of 10 open-ended questions, delivered by the researcher, referring to the impact the intervention had on the participant's quality of life, using a social validity approach.

\section{Follow-up and Outcomes}

The participant was assessed at baseline, 4, 8, and 12 weeks. Adherence to exercise during chemotherapy infusions was $100 \%$, with compliance to exercise intensity reduced only during week 6 (100 bpm vs. usual $124 \mathrm{bpm}$ ). This was due to use of an unfamiliar bicycle. Adherence to the supervised exercise sessions was 94\% (15/16 sessions). Reason for missed session: on holiday. Average compliance to the supervised sessions varied from non-chemotherapy to chemotherapy weeks at 100 and $68.67 \%$, respectively. Performance intensity reductions during chemotherapy weeks were due to nausea. No adverse events from exercise. The participant experienced the following toxicities: grade 2 constipation, grade 2 nausea and vomiting, and grade 2 mucositis. These were managed successfully and did not result in treatment delays or dose attenuations.

With regards to exercise effects, there was a positive change in body composition from baseline to end of the training period, with a decrease in body fat percentage and increase in lean and total body mass (Table 1). In addition to altering body composition, exercise training 


\section{Case Reports in Oncology}

Case Rep Oncol 2019;12:845-854

DOI: $10.1159 / 000503815$

C 2019 The Author(s). Published by S. Karger AG, Basel www.karger.com/cro

McLaughlin et al.: Case Report of Exercise to Attenuate Side Effects of Treatment for Pancreatic Cancer

also induced increases in strength (Fig. 1). Furthermore, physical performance and aerobic capacity improved considerably with exercise (Fig. 2).

With regards to psychological well-being, there were considerable improvements in quality of life, fatigue, and psychological distress, with sleep quality also showing a slight increase with exercise (Fig. 3). In addition to these results, qualitative feedback was obtained from the patient and is outlined in Table 2 .

After 6 weeks of chemotherapy ( 3 cycles), CT scan revealed partial tumour response, with further partial response on 12-week scan (after cycle 6), including improvement in previously noted vascular involvement of his locally advanced pancreatic cancer. Given the improvement, a successful Whipple's pancreatico-duodenectomy was performed without requirement for a vascular reconstruction in September (final pathology: T2 N1 R1). The participant recovered well post-operatively and completed a further 6 cycles post-operative modified FOLFIRINOX (with reduced dose of irinotecan to $150 \mathrm{mg} / \mathrm{m}^{2}$ as per the adjuvant PRODIGE-24 trial protocol [7]). Cycle 12 was delivered with a further $20 \%$ dose reduction of $5-\mathrm{FU}$, oxaliplatin and irinotecan due to grade 2 diarrhoea with cycle 11 . He is now recovering well from his chemotherapy. His Ca19-9 tumour marker remains within normal range and he is planning his return to work.

\section{Discussion}

Exercise was safe during chemotherapy for stage 3 pancreatic cancer, with appropriate attenuation of program when feeling nauseas. Twelve weeks of exercise increased lean muscle mass by $4.4 \%$, translating into increased strength of up to $50 \%$ from baseline to week 12 . These are significant findings as cancer cachexia affects $83 \%$ of those with pancreatic cancer, responsible for $\sim 80 \%$ of advanced pancreatic cancer mortality [8]. Aerobic capacity also increased with training, similar to the previous case study in pancreatic cancer [6]. Improvements in both cardiovascular function and strength are translated into improved physical function, important for maintaining independence of activities of daily living. Usually, chemotherapy results in fatigue sub-scores on FACT-Hep reducing from 1.30 at baseline to 0.80 at 4 and 8 weeks in those with pancreatic cancer [3] due to cachexia, loss of physical function, and psychological distress due to treatment toxicity and tumour-specific cytokine release [9]. This case had large and progressive improvements in fatigue. This is an important finding as cancer-related fatigue upsets daily routines of $88 \%$ of sufferers, with $75 \%$ of those changing employment status [10]. Hence, exercise may be a therapy which can aid maintenance of normal lifestyle by counteracting the usual expected side effects of cachexia and declines in physical functioning.

It is important to discuss the finding from week 6 whereby the participant reported feeling physically and mentally poorly after exercising at a lesser intensity during chemotherapy infusion. It may be speculated that, due to increased side effects from his chemotherapy, the case was too fatigued to exercise to the same intensity. It could be equally speculated that exercise during infusion may have to be at a high intensity to reduce treatment-related side effects. The mechanism suggested for this is redirection of blood flow to muscle tissue during exercise, reducing the splanchnic organ contact with the chemotherapy treatment [11]. This enhances treatment tolerance and is likely to contribute to treatments being on time. Addi- 


\section{Case Reports in Oncology}

Case Rep Oncol 2019;12:845-854

DOI: $10.1159 / 000503815$

C 2019 The Author(s). Published by S. Karger AG, Base www.karger.com/cro

McLaughlin et al.: Case Report of Exercise to Attenuate Side Effects of Treatment for Pancreatic Cancer

tionally, exercise may increase treatment efficacy by increasing tumour blood flow and subsequent chemotherapy delivery. The tumour vasculature does not respond to vasoconstrictive signals during exercise, allowing increased blood flow to the tumour [11], with a $140 \%$ increase in tumour perfusion in breast cancer mice, translating to reduced tumour hypoxia and decreased tumour development, compared with usual care [12]. This may be because hypoxia causes chemoresistance as low vascularization limits the delivery of chemotherapy to the tumor [13]. Therefore, decreased tumour development is postulated to be due to an exercise-induced increase in efficacy of chemotherapy by increasing tumour blood supply.

Additionally, sleep quality also improved by the end of the intervention; and psychological distress moved from 'severe mental disorder' to 'mild mental disorder' from baseline to 4 weeks, and 'likely to be well' by week 8 . Overall, well-being improved, with increased strength, fitness, physical functioning, and improved ability to psychologically cope with treatment, translating to improved QOL. The inclusion of qualitative data adds social validity to the numerical findings, and from Table 2 it can be determined that exercise is truly meaningful to the participant's life. Additionally, exercise during infusion gives the participant 'something to look forward to' and a perception of control (Table 2). This is consistent with a previous study of exercise during infusion whereby it was found that cycling during infusion was " $4 \%$ more comfortable, 15\% less difficult, and 69\% less boring" than usual care [14]. Hence, reduced side effects of treatment by cycling during infusion may be both psychological and physiological. However, it is important to note that he was also seeing a psychologist regularly throughout his chemotherapy, which will have undoubtedly contributed to improved psychological wellbeing.

Unfortunately, cause-effect cannot be determined. The lack of reported treatment side effects may be due to high levels of exercise; or it may be the limited side effects which allows compliance to the intervention. Achieving high levels of exercise may not be possible for those experiencing severe side effects. Moreover, the case is relatively young, compared to the average age of 71 years for diagnosis of pancreatic cancer [15] and hence, results may not be applicable to all pancreatic cancer patients.

\section{Conclusions}

Exercise is safe and effective during treatment for locally advanced pancreatic cancer. This case study has confirmed findings that exercise attenuates expected declines in pancreatic cancer patients, adding to literature that exercising during infusions is also safe and may decrease side effects and aid efficacy of chemotherapy treatment. Findings have clinical implications, and it is recommended that a supervised exercise program and exercise during chemotherapy infusions should be included as an adjunct to usual treatment for pancreatic cancer. In the future, clinical trials of supervised exercise programs are required to expand these findings. 


\section{Statement of Ethics}

Ethical approval was granted from Edinburgh Napier University to undertake this study. The patient gave their informed consent for the publication of this study.

\section{Disclosure Statement}

The authors have no conflicts of interest to declare.

\section{References}

1 Cancer Research UK. Pancreatic cancer statistics. Cancer Research UK. 2015. https://www.cancerresearchuk.org/health-professional/cancer-statistics/statistics-by-cancertype/pancreatic-cancer [accessed 10 December 2018].

2 Hartvig P, Aulin J, Hugerth M, Wallenberg S, Wagenius G. Fatigue in cancer patients treated with cytotoxic drugs. J Oncol Pharm Pract. 2006 Sep;12(3):155-64.

3 Sun V, Ferrell B, Juarez G, Wagman LD, Yen Y, Chung V. Symptom concerns and quality of life in hepatobiliary cancers. Oncol Nurs Forum. 2008 May;35(3):E45-52.

4 Clark KL, Loscalzo M, Trask PC, Zabora J, Philip EJ. Psychological distress in patients with pancreatic cancer-an understudied group. Psychooncology. 2010 Dec;19(12):1313-20.

5 Palesh OG, Roscoe JA, Mustian KM, Roth T, Savard J, Ancoli-Israel S, et al. Prevalence, demographics, and psychological associations of sleep disruption in patients with cancer: University of Rochester Cancer Center-Community Clinical Oncology Program. J Clin Oncol. 2010 Jan;28(2):292-8.

6 Cormie P, Spry N, Jasas K, Johansson M, Yusoff IF, Newton RU, et al. Exercise as medicine in the management of pancreatic cancer: a case study. Med Sci Sports Exerc. 2014 Apr;46(4):664-70.

7 Conroy T, Hammel P, Hebbar M, Ben Abdelghani M, Wei AC, Raoul JL, et al. FOLFIRINOX or gemcitabine as adjuvant therapy for pancreatic cancer. N Engl J Med. 2018 Dec 20;379(25):2395-2406.

8 Bachmann J, Heiligensetzer M, Krakowski-Roosen H, Büchler MW, Friess H, Martignoni ME. Cachexia worsens prognosis in patients with resectable pancreatic cancer. J Gastrointest Surg. 2008 Jul;12(7):1193201.

9 Stewart GD, Skipworth RJ, Fearon KC. Cancer cachexia and fatigue. Clin Med (Lond). 2006 MarApr;6(2):140-3.

10 Curt GA, Breitbart W, Cella D, Groopman JE, Horning SJ, Itri LM, et al. Impact of cancer-related fatigue on the lives of patients: new findings from the Fatigue Coalition. Oncologist. 2000;5(5):353-60.

11 Wiggins JM, Opoku-Acheampong AB, Baumfalk DR, Siemann DW, Behnke BJ. Exercise and the Tumor Microenvironment: Potential Therapeutic Implications. Exerc Sport Sci Rev. 2018 Jan;46(1):56-64.

12 Betof AS, Lascola CD, Weitzel D, Landon C, Scarbrough PM, Devi GR, Palmer G, Jones LW, Dewhirst MW. Modulation of murine breast tumor vascularity, hypoxia, and chemotherapeutic response by exercise. J Natl Cancer Inst. 2015 Mar 16;107(5):djv040.

13 Dewhirst MW, Secomb TW. Transport of drugs from blood vessels to tumour tissue. Nat Rev Cancer. 2017 Dec;17(12):738-50.

14 Edwards KM, Thomas V, Seet-Lee C, Cheema BS, Boyer M, Marthick M. Piloting the Effect of Aerobic Exercise during Chemotherapy Infusion in Patients with Cancer. Med Sci Sports Exerc. 2018;50(5):383-4.

15 Howlader N, Noone AM, Krapcho M, Garshell J, Neyman N, Altekruse SF, et al. SEER Cancer Statistics Review, 1975-2010. 2014. http://seer.cancer.gov/csr/1975_2010/[accessed 10 December 2018]. 


\section{Case Reports in Oncology}

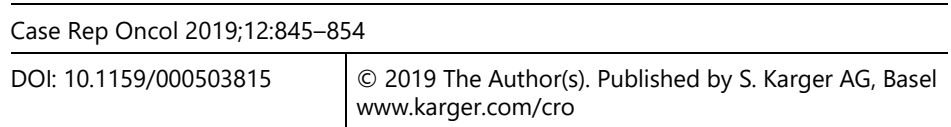

McLaughlin et al.: Case Report of Exercise to Attenuate Side Effects of Treatment for Pancreatic Cancer

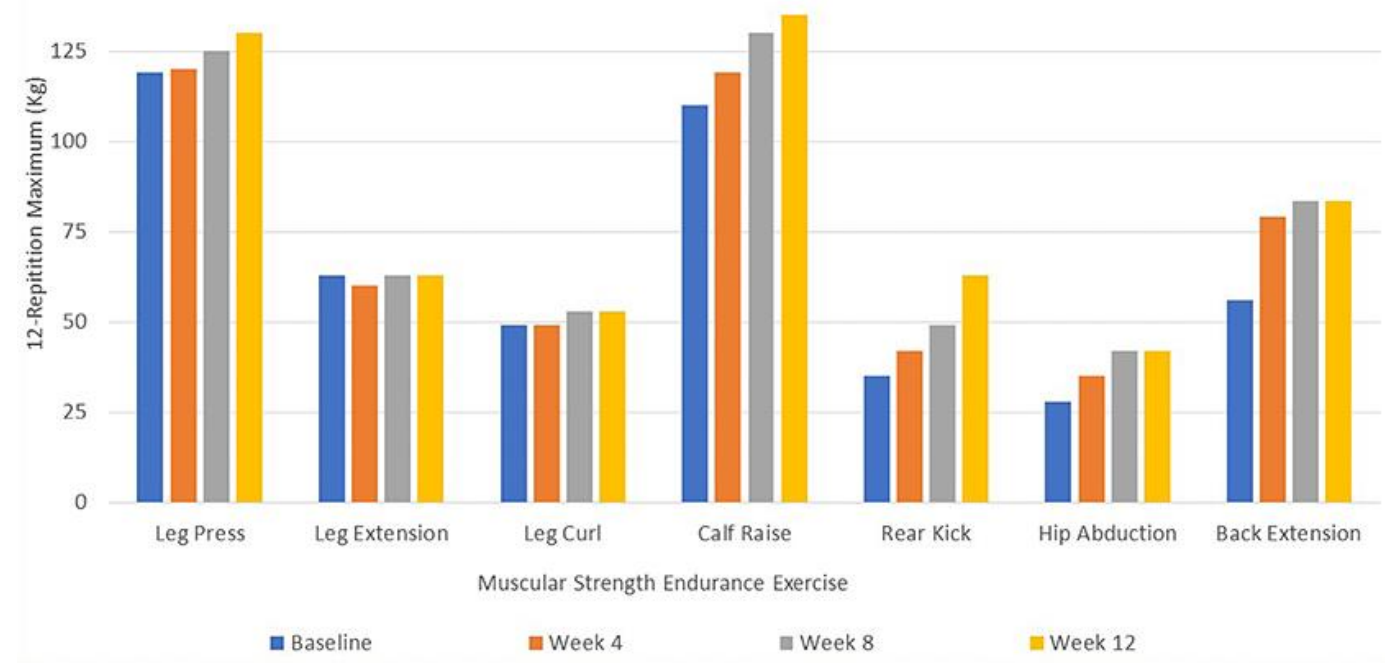

Fig. 1. Muscular strength endurance of leg press, extension and curl, calf raise, rear kick, hip abduction and back extension, measured by 12-repetition maximum (kg), at $0,4,8$, and 12 weeks of the exercise intervention. Results were taken from a single test of each exercise.

20

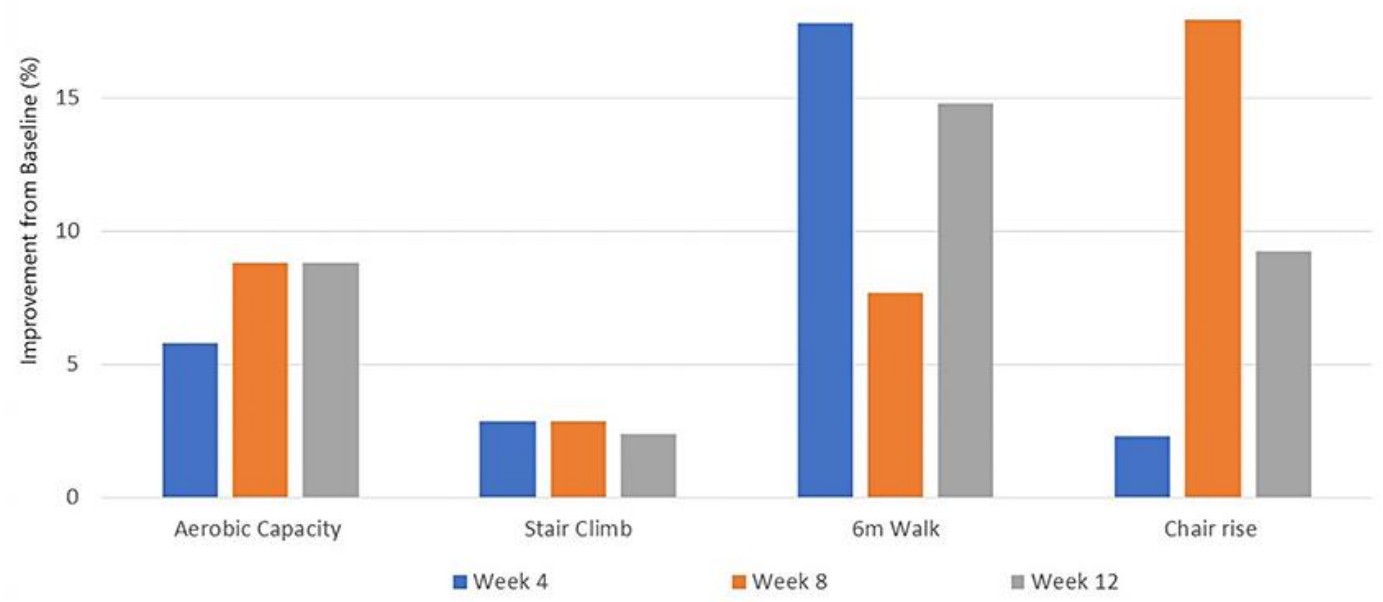

Fig. 2. Physical performance and fitness, measured by a submaximal Astrand fitness test, stair climb time, $6 \mathrm{~m}$ walk test and $30 \mathrm{~s}$ chair rise test, reported as the improvement (\%) from baseline to week 4, 8, and 12 of the exercise intervention. Measurements were taken on 3 consecutive attempts and the average score reported (except from Astrand test, which was performed once, and the result reported). 


\section{Case Reports in Oncology}

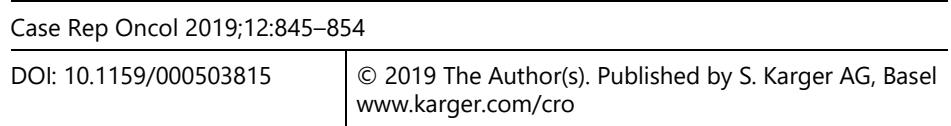

McLaughlin et al.: Case Report of Exercise to Attenuate Side Effects of Treatment for Pancreatic Cancer

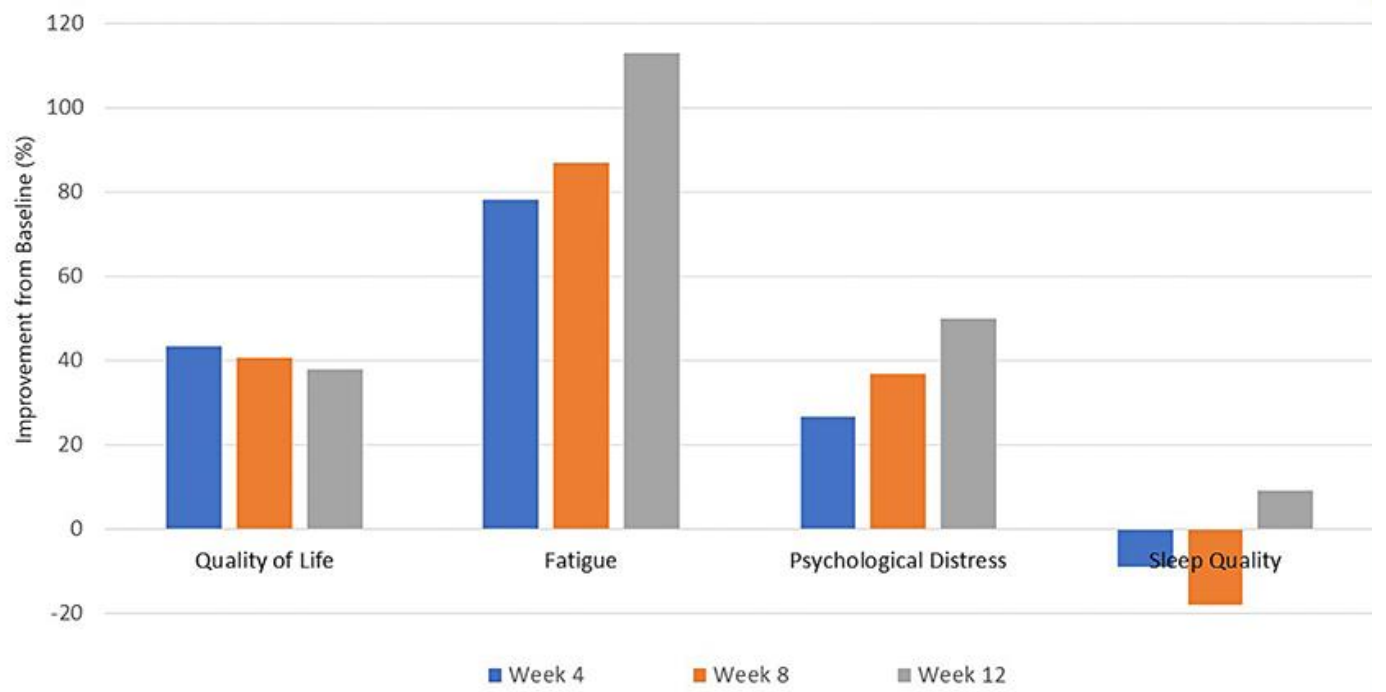

Fig. 3. Psychological well-being, measured by self-reported questionnaires for quality of life, fatigue, psychological distress and sleep quality, reported as the improvement (\%) from baseline to week 4, 8, and 12 of the intervention.

Table 1. Body composition at baseline and week 12

\begin{tabular}{lll}
\hline Measurement & Baseline & Week 12 \\
\hline Body mass, kg & 80.8 & 82.7 \\
Body fat, \% & 17.7 & 13.3 \\
Lean mass, \% & 82.3 & 86.7 \\
\hline
\end{tabular}


Table 2. Semi-structured interview results

\begin{tabular}{ll}
\hline Question & Key Words \\
$\begin{array}{ll}\text { How has exercise helped you cope with } \\
\text { treatment? }\end{array}$ & $\begin{array}{l}\text { Feel different to other cancer patients } \\
\text { In control } \\
\text { Proud }\end{array}$ \\
\hline $\begin{array}{l}\text { What are your main motivators for doing } \\
\text { this exercise programme? }\end{array}$ & Fear of death \\
\hline $\begin{array}{l}\text { How would you describe your experience } \\
\text { of this programme? }\end{array}$ & $\begin{array}{l}\text { Hopeful } \\
\text { Purposeful } \\
\text { Team effort (social) } \\
\text { Don't feel isolated }\end{array}$ \\
\hline $\begin{array}{l}\text { Have any side effects of treatment been a } \\
\text { problem for exercising? }\end{array}$ & $\begin{array}{l}\text { Nausea a big problem in first week } \\
\text { Frustrating }\end{array}$ \\
$\begin{array}{l}\text { What do you think are the benefits of } \\
\text { cycling during infusion? }\end{array}$ & $\begin{array}{l}\text { Something to look forward to } \\
\text { Not easy but makes a big difference } \\
\text { Helps to aid recovery from treatment: Felt } \\
\text { awful for a full week when bike was stolen but } \\
\text { all other times recovered after a few days }\end{array}$ \\
\hline
\end{tabular}

